\title{
MULTI-TARGET MOLECULE TO TREAT DIABETIC NEPHROPATHY IN RATS
}

\author{
Md Abdul Khan ${ }^{1}$, Sung Hee Hwang ${ }^{2}$, Scott Barnett ${ }^{1}$, Anna Burkhan ${ }^{1}$, Wojciech \\ Jankiewicz ${ }^{1}$, Bruce Hammock ${ }^{2}$, and John Imig ${ }^{1}$ \\ ${ }^{1}$ Medical College of Wisconsin \\ ${ }^{2}$ University of California
}

September 14, 2020

\begin{abstract}
Background and Purpose: Diabetic nephropathy is one of the most common complications that is related to high morbidity and mortality in type 2 diabetic patients. We investigated ability of a novel dual modulator, PTUPB that concurrently acts as a soluble epoxide hydrolase inhibitor and as a cyclooxygenase-2 inhibitor against diabetic nephropathy. Experimental Approach: Sixteen-week-old type 2 diabetic and proteinuric obese ZSF1 rats were orally treated with vehicle, PTUPB, or enalapril for 8 weeks. Key Results: PTUPB alleviated diabetic nephropathy in obese ZSF1 rats by reducing albuminuria by $50 \%$, renal tubular cast formation by $60-70 \%$, renal fibrosis by $40-50 \%$, glomerular injury by $55 \%$ and preserved glomerular nephrin expression. Enalapril demonstrated comparable effects and alleviated diabetic nephropathy in obese ZSF1 rats by reducing all kidney injury parameters by 30 to $50 \%$. Diabetic renal injury in obese ZSF1 rats was accompanied by renal inflammation with 6-7-fold higher urinary MCP-1 level and renal infiltration of CD-68 positive cells. PTUPB and enalapril reduced renal inflammation but PTUPB demonstrated superior anti-inflammatory actions than enalapril. Obese ZSF1 rats were also hypertensive, hyperlipidemic, and exhibited liver injury. Interestingly, PTUPB but not enalapril decreased hyperlipidemia and liver injury in Obese ZSF1 rats. Conclusion and Implication: Overall, we demonstrate that a dual modulator PTUPB does not treat hyperglycemia, but can effectively alleviate hypertension, diabetic nephropathy, hyperlipidemia, and liver injury in type 2 diabetic rats. Therefore, we suggest that PTUPB has promising potential to be developed as a novel therapy for type 2 diabetic nephropathy and other complications.
\end{abstract}

\section{What is already known?}

Diabetic nephropathy is the leading cause of death and disability in type 2 diabetic patients. Complex pathophysiology of diabetic nephropathy makes it difficult to treat and often requires multiple treatment approaches.

\section{What this study adds?}

We demonstrate strong pre-clinical therapeutic efficacy of a novel bifunctional molecule to treat diabetic nephropathy and other diabetic complications.

\section{What is the clinical significance?}

The multi-target drug PTUPB has compelling potential to treat type 2 diabetic complications.

\section{INTRODUCTION}

Diabetes is a significant health problem worldwide. Globally $8.5 \%$ of adults aged 18 years and older have diabetes and diabetes was the direct cause of 1.6 million deaths in 2016. The high mortality and morbidity associated with diabetes is due to several complications. One of the most common diabetic complications 
is micro- and macro-vascular dysfunction that results in diabetic nephropathy that progresses to end-stage renal diseases (ESRD) and death (Warren et al., 2019). Approximately 20-40\% of diabetic patients develop nephropathy, and due to the growing incidence of diabetes, diabetic nephropathy is now the main cause of ESRD worldwide (Toth-Manikowski and Atta, 2015). Moreover, diabetic nephropathy incidence rates show no signs of slowing. In the USA alone, $42 \%$ of all ESRD cases had a diagnosis of diabetic nephropathy (Warren et al., 2019).

Besides glycemic control with anti-diabetic drugs, renin angiotensin system (RAS) blockers have been the standard of care to treat diabetic nephropathy. Unfortunately, approximately one-half of diabetic patients fail to achieve acceptable glycemic control with the currently available anti-diabetic options and RAS blockers have been mildly effective in reducing diabetic nephropathy progression to ESRD. Consequently, morbidity and mortality associated with diabetes is still high because of complications such as hypertension and diabetic nephropathy (Catalá-López et al., 2016; Delanaye and Scheen, 2019). The poor treatment outcome with the present anti-diabetic drugs is also partly due to uncontrolled comorbid risk factors such as dyslipidemia in type 2 diabetes (Patti et al., 2020). Indeed, according to the American Diabetic Association, during the period of 2009-2012 about 65-70\% of diabetic patients had pre-existing dyslipidemia and other complications at the time of diagnosis (Afkarian et al., 2013). Thus, there is an urgent need for novel treatments that reduce cardiovascular risk, have a favorable impact on comorbid conditions such as diabetic nephropathy. We suggest that any such novel treatment approach that will target multiple pathologies associated with type 2 diabetes can be molecule with multiple pharmacophores, possibly a bifunctional molecule.

The concept that bifunctional molecules could be developed to treat diseases has been energized by the approval of Entresto, a combined Neprilysin and Angiotensin Type 1 Receptors inhibitor, for heart failure (Chalikias et al. 2020). These multi-target drugs have much more potential than single-target and highly specific agents due to better $(i)$ disease modifying actions, $(i i)$ additive and/or synergistic therapeutic actions, (iii ) more predictable pharmacokinetics, ( $i v)$ prolonged duration of effectiveness, and $(v)$ lower probability for drug interactions. As a result, it is increasingly recognized that a balanced modulation of two targets can provide a superior therapeutic effect and milder side effect profile.

We developed a bifunctional molecule that concurrently inhibits sEH and COX-2. Inhibitor of sEH possess anti-hypertensive, anti-diabetic, anti-inflammatory, and anti-fibrotic actions (Imig, 2018). COX-2 selective inhibitors are widely used to manage inflammation by inhibiting the generation of pro-inflammatory COX metabolites (Nandakishore et al., 2014). Combining sEH inhibition with COX-2 inhibition can limit COX-2 side effects while increasing therapeutic potential to combat organ damage. Moreover, we have found that coadministration of lower doses of COX-2 inhibitors with a sEH inhibitor reduces the potential risk of adverse cardiovascular events (Grosser et al., 2006). Thus, the benefits of each agent in this combination are retained and the mechanism-based adverse effects are attenuated. This synergy provides support to the concept that as a single molecule such as the sEH/COX-2 inhibitor, PTUPB, has therapeutic potential for complex chronic diseases while decreasing the side effects associated with COX-2 inhibitors. In the present study, we investigated therapeutic potentials of PTUPB in a type 2 diabetic nephropathy model and demonstrated its promising effects.

\section{MATERIALs AND METHODs}

\subsection{Chemicals}

The chemistry and synthesis process for the dual sEH and COX-2 inhibitor PTUPB is described previously (Hwang et al., 2011). All chemicals used in this study were purchased from Sigma Aldrich (St Louis, MO, USA) unless otherwise mentioned.

\subsection{Animal studies}

All experiments are approved by the Medical College of Wisconsin Institutional Animal Care and Use Committee that concurs with the National Institutes of Health Guidelines. In the Biomedical Resource Center at the Medical College of Wisconsin the rats were housed with a $12 / 12 \mathrm{~h}$ light-dark cycle with free 
access to water and rat chow.

\subsubsection{Experimental design}

These studies were carried out in 16 week-old, male obese ZSF1 rats (ZSF1-Lepr ${ }^{\text {fa }}$ Lepr $^{\text {cp }} /$ Crl; strain code 378) and lean ZSF1 rats (strain code 379) obtained from Charles River Laboratories. The ZSF1 rat is a cross between Zucker Diabetic Fatty (ZDF) female and Spontaneously Hypertensive Heart Failure (SHHF) male rats. These rats are known to be an excellent model of diabetic nephropathy (Bilan et al., 2011).

As we have described in a recent study, at 16 weeks of age the obese ZSF1 rats were diabetic, hypertensive, and had renal injury compared to the age-matched lean ZSF1 rats (Hye Khan et al., 2018). Diabetes (plasma glucose $>250 \mathrm{mg} / \mathrm{dL}$ ), hypertension $(\mathrm{SBP}>150 \mathrm{mmHg}$ ), and proteinuria $(>150 \mathrm{mg} / \mathrm{d})$ were confirmed in the obese ZSF1 rats. The rats were randomized into four groups ( $n=6-8 /$ group), Group 1: Lean ZSF1 rats treated with vehicle; Group 2: obese ZSF1 rats treated with vehicle; Group 3: obese ZSF1 rats treated with PTUPB (10mg/kg/d i.p.), and Group 4: obese ZSF1 rats treated with enalapril (10mg/kg/d p.o.). PTUPB administration was carried out using an intra-peritoneal implanted osmotic pump (ALZET ${ }^{\circledR}$ osmotic pump, DURECT Corporation,Cupertino, CA). All rats were weighed, and systolic blood pressure was measured by tail-cuff plethysmography (IITC Life Science Inc., Woodland Hills, CA, USA) during the 8 week treatment protocol.

At baseline, intraperitoneal glucose tolerance tests, blood pressure measurements, and urine collections were conducted. At the end of the 8 week treatment protocol parameters were measured and rats were euthanized for plasma and tissue collection. Urine, blood, plasma, kidney, and liver samples were frozen and stored at $-80^{0} \mathrm{C}$ for biochemical assays and other analysis. The kidney and liver tissue samples were either immersion fixed in 10\% neutral buffered formalin for paraffin embedding or processed for frozen section for use in histopathology and immunohistopathological experiments.

\subsection{Glucose tolerance test}

Intra-peritoneal glucose tolerance test was carried out at baseline and at the end of the 8-week treatment protocol. After a fasting blood sample collection, glucose ( $2 \mathrm{~g} / \mathrm{kg}$ i.p.) was injected in fasted rats (8-12 hours) followed by tail vein blood sampling at different time points. Blood glucose levels were measured using a glucometer LifeScan (Miltipas, CA, USA).

\subsection{Biochemical assays}

The glycemic status of the rats was determined by measuring hemoglobin A1C (HbA1c) levels in whole blood using a biochemical assay from Crystal Chem and by measuring plasma insulin using ELISA (Mercodia AB, Uppsala, Sweden). Lipid profile was determined by measuring plasma cholesterol, triglyceride, and non-esterified free fatty acid (NEFA) levels using assay kits from Cayman Chemical (Ann Arbor, MI, USA). Plasma low-density lipoprotein (LDL) was measured using a kit from Crystal Chem (Downers Grove, IL, USA). Homeostatic model assessment (HOMA-IR) was carried out to determine insulin sensitivity in experimental groups. HOMA-IR was calculated from fasting blood glucose. Liver enzymes aspartate aminotransferase (AST) and alanine aminotransferase (ALT) were measured using commercial assay kits (Sigma Millipore, St. Louis, MO, USA). Urinary protein and creatinine were measured calorimetrically using assay kits from Cayman Chemical. Urinary levels of albumin and monocyte chemoattractant protein-1 (MCP-1) were determined using ELISAs from Exocell (Philadelphia, PA, USA) and BD Biosciences (San Jose, CA, USA), respectively.

\subsection{Histopathological analysis}

Kidney and liver tissues were formalin fixed and paraffin embedded. The paraffin embedded tissues were cut into $4 \mu \mathrm{m}$ sections for histological analysis. Tissue sections were deparaffinized, re-hydrated, and kidney tissue slices were stained with Periodic Acid-Schiff (PAS) and Picrosirius Red (PSR). Glomerulosclerosis and mesangial matrix expansion were scored from kidney sections stained with PAS staining using methods described earlier (Hye Khan et al., 2018,2019). Histological analysis was done at a magnification of 400X 
to assess glomerular injury, and renal tubular proteinaceous cast was assessed at a magnification of 200X using Nikon NIS Elements Software (Nikon Instruments Inc., Melville, NY, USA). The percentage area positive for proteinaceous cast was calculated from the mean of eight cortical and five medullary fields for each animal. Fibrosis in the kidney and liver were determined from kidney and liver sections stained with PSR and examined at a magnification of 200X. In the kidney, the percentage area positive for collagen was calculated as the fibrotic area from the mean of eight cortical and five medullary fields for each kidney sample. The mean percentage of collagen positive liver fibrotic areas were calculated from 20 fields in each liver sample. In assessing liver steatosis, frozen liver sections $(10 \mu \mathrm{m})$ were stained with Oil Red O dye according to manufacturer's protocol (Abcam, Cambridge, MA, USA). The percent of liver tissue area with lipid accumulation was calculated as described earlier (Hye Khan et al., 2018). The percentage area positive for lipid accumulation was calculated from the mean of 20 fields (at 200X magnification) for each animal using Nikon NIS Elements Software. Histological scoring was carried out by two observers who were blinded to the identity of the experimental groups.

\subsection{Immunohistopathological analysis}

Immune cell infiltration in the kidney was determined using immunohistopathological analysis. Tissue sections were incubated with rodent declocker solution (Biocare Medical, Concord, CA, USA) at $95^{\circ} \mathrm{C}$ for antigen retrieval (Hye Khan et al., 2016). Kidney sections were immunostained with anti-CD68 (1:100; Serotec, Raleigh, NC, USA) to determine renal macrophage/monocyte infiltration. Biotinylated rat anti-mouse secondary antibody (1:200) was used for development with avidin-biotinylated HRP complex (Vectastain ABC Elite kit, Vector Laboratories, Burlingame, CA, USA) followed by hematoxylin counterstaining. Stained tissue sections were examined by light microscopy (400x magnification) and digital images were taken for analysis using Nikon NIS Elements Software. Kidney macrophage/monocyte infiltration was determined by counting CD-68 positive cells. As described earlier, (Hye Khan et al., 2016) the number of positive cells per field was divided by the metric area of the field to obtain the number of positive cells per $\mathrm{mm}^{2}$. All immunohistopathological analysis were done in blinded fashion by two observers.

\subsection{Immunofluorescence analysis}

Formalin formalin-fixed and paraffin-embedded kidney sections $(4 \mu \mathrm{m})$ were de-paraffinized, re-hydrated, and incubated with rodent declocker solution (Biocare Medical, Concord, CA, USA) at $95^{\circ} \mathrm{C}$ for antigen retrieval. Kidney sections were then immunostained with anti-nephrin (1:100; Santa Cruz Biotechnology, Inc, Dallas, TX, USA) to determine renal expression of nephrin. Donkey anti-rabbit IgG H\&L (Alexa Fluor@ 488) secondary antibody (1:200; Abcam, Cambridge, MA, USA) was used for development with fluorescence quenching liquid (Vector Laboratories, Burlingame, CA, USA). Immunostained sections were examined by Nikon 55i fluorescence with a green excitation (200x magnification) and digital images were taken for analysis using Nikon NIS Elements Software. Nephrin expression in the kidney was determined by measuring the percentage of nephrin positive kidney areas. Two observers blinded to the identity of the samples conducted nephrin image analysis in kidney sections.

\subsection{Determination of glomerular albumin permeability}

Glomeruli from 8-10 week Sprague Dawley rats were isolated as previously described (Ilatovskaya, 2015) using a variable-sieving process $(150 \mu \mathrm{m}, 106 \mu \mathrm{m}$, then $75 \mu \mathrm{m})$ after an in-vivo renal perfusion of high-molecular weight FITC-labeled dextran $(150 \mathrm{kD}$, TdB Consultancy AB, Uppsala, Sweden). Each condition was tested with 3 or more rats, and a minimum of 9 glomeruli. Glomeruli were affixed to the optical window of poly-L-lysine coated culture dish (MatTek, Ashland, MA), and bathed in a 5\% BSA solution containing (in $\mathrm{mM}$ ): $145 \mathrm{NaCl}, 2 \mathrm{CaCl} 2,4.5 \mathrm{KCl}, 2 \mathrm{MgCl} 2,10 \mathrm{HEPES}$, TRITC-labeled dextran, pH 7.35 (adjusted with $\mathrm{NaOH}$ ). Only de-encapsulated glomeruli with detached afferent and efferent arteriole were included. Using an AR-1 confocal microscope, a Z-stack of 27 images (total thickness of $72 \mu \mathrm{m}$ ) was collected while in the initial $5 \%$ solution (baseline), which was followed by a repeated collected every 2 min following bath exchange to $1 \%$ albumin bath solution, for a total of 10 minutes. Glomeruli were either pre-incubated for 5 minutes in angiotensin II $(10 \mu \mathrm{M})$ to induce permeability in the presence and absence of PTUPB $(1 \mu \mathrm{M})$ to 
determine its effects on permeability. Following the conclusion of the experiment, volumetric recompositing of individual z-planes was carried out using Fiji image processing package (ImageJ 2.0.0, National Institutes of Health, USA) and Origin Pro 6.0 (Origin Lab, Northampton, MA).

\subsection{Statistical analysis}

Data are reported as box and whisker plots with median, minimum to maximum, and 10 to 90 percentiles. Figure $4 \mathrm{E}$ is plotted as mean \pm S.E.M. The statistical significance between two groups was determined by a two-tailed unpaired Student's t test (and among more than two groups it was determined by repeated measure one-way analysis of variance followed by Tukey's post-hoc test) using GraphPad Prism ${ }^{\circledR}$ Version 4.0 software (GraphPad Software Inc, La Jolla, CA, USA). Difference between two groups was considered significant when probability values of $\mathrm{P}$ were less or equal to 0.05 .

\section{RESULTS}

\subsection{PTUPB reduces blood pressure and lacks anti-diabetic actions in obese ZSF1 rats}

Obese ZSF1 rats were severely diabetic with 60 to $75 \%$ higher fasting blood glucose, increased HbA1c, increased HOMA index, decreased rate of glucose clearance, and marked hyperinsulinemia compared to lean ZSF1 rats (Figure 1). Neither interventional PTUPB nor enalapril treatment attenuated hyperglycemia, hyperinsulinemia, and insulin resistance in obese ZSF1 rats. All markers of overt type 2 diabetes remained markedly elevated compared to lean ZSF1 rats. The obese ZSF1 rats had increased body weight (733 $\pm 11 \mathrm{~g})$ compared to lean ZSF1 rats $(470 \pm 9 \mathrm{~g})$, and neither PTUPB $(702 \pm 7 \mathrm{~g})$ nor enalapril $(722 \pm 10 \mathrm{~g})$ treatments affected body weight of obese ZSF1 rats. Systolic blood pressure was higher in obese ZSF1 rats (182 \pm 7 $\mathrm{mmHg})$ compared to lean ZSF1 rats $(139 \pm 5 \mathrm{mmHg})$. In obese ZSF1 rats 8-week PTUPB and enalapril treatments reduced blood pressure. Blood pressure averaged $161 \pm 9 \mathrm{mmHg}$ in PTUPB and averaged $147 \pm 10$ $\mathrm{mmHg}$ in enalapril treated obese ZSF1 rats.

\subsection{PTUPB improves plasma lipids in obese ZSF1 rats}

Plasma cholesterol, triglycerides, NEFA, and LDL-cholesterol were 70-90\% higher in obese ZSF1 rats compared to lean ZSF1 rats. Interventional PTUPB treatment markedly improved the lipid profile of obese ZSF1 rats and reduced plasma lipids to levels similar to lean ZSF1 rats. Interestingly, enalapril treatment did not affect the lipid profile of obese ZSF1 rats and the plasma lipid levels remained elevated in obese ZSF1 rats (Figure 2).

\subsection{PTUPB reduces renal injury in type 2 diabetic obese ZSF1 rats}

Obese ZSF1 rats demonstrated marked renal injury compared to lean ZSF1 rats. In obese ZSF1 rats, albuminuria was more than 40 fold higher compared to lean ZSF1 rats. Obese ZSF1 rats had 4 to14-fold higher renal cortical and medullary cast area compared to lean ZSF1 rats. Interventional PTUPB treatment markedly reduced kidney injury with albuminuria reduced by $40 \%$ and tubular cast formation reduced by $60 \%$ compared to vehicle obese ZSF1 rats. Enalapril also attenuated kidney injury and exhibited an equipotent effect as PTUPB in attenuating albuminuria, while it was relatively weaker in attenuating renal tubular cast formation in obese ZSF1 rats (Figure 3A-D).

Obese ZSF1 rats demonstrated marked glomerular injury with a glomerular injury score that was $80 \%$ higher than lean ZSF1 rats. Both interventional PTUPB and enalapril treatments equipotently attenuated glomerular injury by $50 \%$ in obese ZSF1 rats (Figure 4 A, C). Consistent with marked glomerular injury, the obese ZSF1 rats had $60 \%$ lower renal nephrin expression compared to lean ZSF1 rats. PTUPB and enalapril treatments equipotently elevated nephrin expression in the kidney of obese ZSF1 rats (Figure 4 B, D). Experiments in isolated glomeruli demonstrate that angiotensin II markedly elevated glomerular albumin permeability. Interestingly, PTUPB directly protected the glomerular filtration barrier and attenuated angiotensin II-induced increase glomerular albumin permeability. In the presence of PTUPB, angiotensin II-induced glomerular albumin permeability was $>50 \%$ lower than angiotensin II alone (Figure 4E). 
Renal fibrosis was $60 \%$ higher in obese ZSF1 rats compared to lean ZSF1 rats. Interventional PTUPB and enalapril treatments demonstrated marked anti-fibrotic actions and equipotently reduced renal collagen deposition in obese ZSF1 rats (Figure 5A-C).

In the present study, marked renal inflammation with elevated urinary MCP-1 excretion and infiltration of renal macrophages was evident in obese ZSF1 rats. Urinary MCP-1 excretion was $80 \%$ higher in obese ZSF1 rats and PTUPB reduced urinary MCP-1 excretion by $53 \%$. Enalapril also demonstrated anti-inflammatory action in obese ZSF1 rats and reduced MCP-1 excretion by 28\%. In obese ZSF1 rats, elevated chemokine expression was accompanied by marked renal infiltration of immune cells, and obese ZSF1 rat kidneys had $80 \%$ higher CD-68 positive immune cells compared to lean ZSF1 rats. PTUPB and enalapril reduced renal infiltration of immune cells in obese ZSF1 rats by 60 and 45\%, respectively (Figure 6A-C). Overall, we demonstrated marked renal injury in type 2 diabetic obese ZSF1 rats. PTUPB and enalapril attenuated renal injury. Interestingly, we demonstrated that PTUPB and enalapril acted similarly with PTUPB being superior in reducing renal tubular injury and inflammation.

\subsection{PTUPB reduces liver injury and steatosis in obese ZSF1 rats}

Type 2 diabetic obese ZSF1 rats had liver injury along with hyperlipidemia. Obese ZSF1 rats had 2-3 fold higher plasma levels of two important liver injury biomarkers, AST and ALT, compared to lean ZSF1 rats. Interventional PTUPB but not enalapril treatment lowered AST and ALT plasma levels to levels similar to that of lean ZSF1 rats (Figure 7A, B). The obese ZSF1 rats had marked liver fibrosis with a 5-fold higher liver collagen expression compared to lean ZSF1 rats. PTUPB treatment markedly reduced liver collagen expression in the obese ZSF1 rats. Enalapril treatment was ineffective in lowering liver fibrosis in obese ZSF1 rats (Figure 7C, E). The obese ZSF1 rats had marked hepatosteatosis with 3 times higher liver steatotic area compared to lean ZSF1 rats. PTUPB treatment attenuated hepatosteatosis in the obese ZSF1 rats by 50\%. Enalapril treatment did not affect hepatosteatosis in obese ZSF1 rats. (Figure 7D, F).

\section{DISCUSSION}

Diabetic nephropathy is a major cause of ESRD worldwide. According to the World Health Organization (WHO), 1 in every 11 adults worldwide are diabetic (Saran et al., 2020). This high incidence of diabetes is linked to the high incidence of diabetic nephropathy and consequent ESRD. Approximately 20-40\% of all diabetic patients develop diabetic kidney disease in their lifetime, which often progresses to ESRD (Isomaa et al., 2001; Grundy, 2006). Unfortunately, despite the recent progress in our understanding, the complicated pathophysiology of diabetic nephropathy has limited our success to treat and manage diabetic nephropathy. Hence, there is growing interest in developing novel therapies that will target multiple pathophysiological factors of type 2 diabetes and its renal complications (Roche et al., 2015).

Over the years, several studies demonstrated critical etio-pathological roles of eicosanoids in the type 2 diabetes and associated kidney disease (Lorthioir et al. 2012; Molinar-Toribio et al., 2015). Indeed, a large number of studies demonstrated that eicosanoid metabolites are associated with type 2 diabetes, blood pressure, lipid levels, and insulin signaling (Imig, 2018; Bellucci et al., 2017; Nasrallah et al., 2016; Harris, 2008). In the present interventional study in overt type 2 diabetic obese ZSF1 rats, we investigated the effect of a novel molecule that concurrently acts on two pathways of arachidonic acid metabolism in type 2 diabetes. Our findings demonstrate that interventional treatment with the dual acting sEH/COX-2 inhibitor, PTUPB, reduces diabetic kidney injury in type 2 diabetic, hypertensive, and hyperlipidemic obese ZSF1 rats.

In type 2 diabetic obese ZSF1 rats, we compared the efficacy of interventional PTUPB treatment with an angiotensin converting enzyme (ACE) inhibitor, enalapril. It is important to note that ACE inhibitors are widely used for blood pressure control and are particularly beneficial in hypertensive type 2 diabetic subjects to treat diabetic nephropathy (Batlle et al., 2012). Interestingly, we found that PTUPB did not reduce hyperglycemia and hyperinsulinemia in obese ZSF1 diabetic rats. This is consistent with the findings of an earlier study where we reported that PTUPB prevents development of type 2 diabetes in Zucker diabetic fatty (ZDF) rats (Hye Khan et al., 2016). PTUPB treatment prevented development of insulin resistance and elevation of blood glucose in ZDF rats. PTUPB treatment was given prior to an elevation 
in blood glucose and insulin resistance to the ZDF rats. Indeed, the objective of that earlier study was to investigate if PTUPB could prevent development of type 2 diabetes. Findings of this previous study prompted us to carry out the present study to investigate the ability of PTUPB interventional treatment to reduce diabetes and its complication in a rat model of severe type 2 diabetes and diabetic nephropathy. Our findings demonstrate that interventional PTUPB treatment failed to reduce blood glucose or improve glucose homeostasis in obese ZSF1 rats. Similar to interventional PTUPB treatment, interventional ACE inhibitor treatment with enalapril did not affect hyperglycemia and insulin resistance in obese ZSF1 rats. These findings are in accord to our earlier findings on the effects of enalapril in obese ZSF1 diabetic rats (Hye Khan et al., 2018). In contrast to our findings, there are studies that demonstrate beneficial enalapril effects on blood glucose and insulin sensitivity. These studies found that enalapril improved insulin sensitivity in fructose-fed spontaneously hypertensive and Cohen Diabetic rats (Vuorinen-Markkola and Yki-Järvinen, 1995). Enalapril is also reported to improve glucose storage and insulin sensitivity in hypertensive type 1 diabetic patients (Rosenthal et al., 1995). The discrepancy between our findings in obese ZSF1 rats and these earlier studies could be due to the different interventional experimental design and the use of different diabetic rat models. Overall, we demonstrate that dual sEH/COX-2 inhibitor PTUPB or enalapril interventional treatment did not reduce type 2 diabetes in obese ZSF1 rats.

An important finding of the present study is the reduction in kidney injury by interventional PTUPB treatment in type 2 diabetic obese ZSF1 rats. The obese ZSF1 rats develop diabetic nephropathy with marked kidney functional and structural injuries (Hye Khan et al., 2018; Bilan et al., 2011; Su et al., 2018). We demonstrate potent renal actions of PTUPB in reducing renal functional and structural injuries in type 2 diabetic obese ZSF1 rats.

The pathophysiology of diabetic nephropathy is complex due to the presence of several comorbid conditions in type 2 diabetic patients. Most often these comorbid conditions are hypertension and hyperlipidemia (Ritz et al., 2001). In clinical studies, it is demonstrated that better blood pressure control in type 2 diabetes decreased the onset or degree of kidney injury and vascular complications (Xie et al., 2016). In the present study, interventional PTUPB treatment demonstrated marked anti-hypertensive actions in obese ZSF1 rats. A similar anti-hypertensive action of PTUPB has been reported in an earlier study (Hye Khan et al., 2016). This anti-hypertensive action of PTUPB could be related to the sEH inhibitor activity. The sEH inhibitors are widely reported to be anti-hypertensive, and this effect has been attributed to its ability to increase the ratio of epoxyeicosatrienoic acids (EETs) to their less biologically active diols (Imig et al., 2002; Neckář et al., 2012). Unlike sEH inhibition, COX-2 inhibition is not anti-hypertensive (Zhao et al., 2005; Cheng and Harris, 2004) and COX-2 inhibitors do not affect blood pressure in humans and animals (Bombardier et al., 2000). However, it reported that COX-2 inhibitor celecoxib increased systolic, diastolic and mean blood pressure in rat with normal blood pressure (Safaeian et al., 2018). Additionally, some clinical studies have shown that chronic COX-2 inhibition can induce hypertension in patients. It is reported that the COX-2 inhibitor Vioxx increase blood pressure in in human and it is considered as a significant adverse effect (Cho et al., 2003). Hence, it is likely that the anti-hypertensive effect of PTUPB in this study is due to sEH inhibitory activity. Moreover, it led us to suggest that use of PTUPB will be beneficial in patients who need COX-2 inhibition without developing COX-2 related adverse effect.

In chronic kidney disease, including diabetic nephropathy, disease progression is associated hyperlipidemia which is a common co-morbid condition of type 2 diabetes (Ferro et al., 2018). Important and beneficial renal outcomes of current lipid lowering therapies are known on complications in type 2 diabetes patients (Cases and Coll, 2005; Ferro et al., 2018). In the present study, interventional PTUPB treatment demonstrated an interesting lipid lowering action in type 2 diabetic obese ZSF1 rats. This lipid lowering action of PTUPB can be attributed to its sEH inhibitory activity as the lipid lowering effect of sEH inhibition has been reported in several studies (EnayetAllah et al., 2008). A polymorphism in the sEH gene (EPHX2) has been reported in humans with marked lipid abnormalities. It is reported that the R287Q variant of sEH is associated with elevated plasma cholesterol and triglycerides in familial hypercholesterolemia (EnayetAllah et al., 2008). Additionally, animal studies in sEH null (EPHX2 -/-) mice demonstrated lower plasma total cholesterol levels and lower HMG-CoA reductase activity (EnayetAllah et al., 2008). A similar lipid lowering action has 
been reported for COX-2 inhibition (Imig et al., 2005). These earlier findings are in accord with our current findings in obese ZSF1 rats and led us to suggest that the marked lipid lowering actions of PTUPB is caused by actions on COX-2 and sEH pathways.

Unlike PTUPB, interventional enalapril treatment did not cause any beneficial effect on lipid profile of obese ZSF1 rats. As reported in an earlier study, it is possible that 12-24 weeks long enalapril treatment could affect lipid profile in obese ZSF1 rats (Bilan et al., 2011). However, it should be noted that in this previous study enalapril treatment was given prior the development of hyperlipidemia. Moreover, it is not yet known if enalapril or any ACE inhibitor can alleviate established hyperlipidemia in a pre-clinical type 2 diabetic nephropathy model like the obese ZSF1 rat.

Elevated renal inflammation is an important pathophysiological factor of diabetic nephropathy. Indeed, chronic inflammation is a hallmark of metabolic dysfunctions including type 2 diabetes, hypertension, and hyperlipidemia. It is reported that the severity of renal inflammation and its renal consequence depends on the presence of different metabolic pathologies (Hotamisligil, 2006; Zhang and Lerman, 2016). During metabolic dysfunctions the normal physiological regulatory system is disrupted and initiates a cascade of deleterious inflammatory responses in multiple organs including the kidney (Hotamisligil, 2006; Furman et al., 2019). During metabolic diseases like type 2 diabetes, infiltration of immune cells and cytokine production occur in the abdominal and peri-renal fat and acts as vital source of inflammation in the kidney (Ma et al., 2006). In the present study, we demonstrate marked renal inflammation with renal macrophage infiltration and elevated chemokine production in obese ZSF1 rats. Similar to this finding, we earlier demonstrated renal injury associated with increased renal chemokine and elevated renal infiltration of immune cells in other metabolic disease models (Hye Khan et al., 2018; Imig et al., 2012). Interestingly, interventional PTUPB treatment reduced renal inflammation by reducing renal infiltration of immune cells and chemokine MCP-1 production in type 2 diabetic obese ZSF1 rats. We also demonstrate that renal inflammation in obese ZSF1 rats was ameliorated by enalapril and this finding is in agreement with the findings of a recent study in same rat model (Hye Khan et al., 2018).

Several recent studies demonstrated marked anti-inflammatory actions of PTUPB in multiple pathological conditions and in multiple organs including the kidney. In a mice sepsis model, PTUPB reduced systemic inflammation and reduced liver and kidney injury (Zhang et al., 2020). Certain chemotherapy drugs cause treatment limiting macrophage driven cytokine surge and PTUPB treatment prevented such cytokine surge during chemotherapy (Gartung et al., 2019). In an earlier study, we demonstrated that PTUPB prevented development of renal inflammation in ZDF rats by preventing macrophage infiltration in the kidney (Hye Khan et al., 2016). In these earlier studies we and others have provided evidence that PTUPB has anti-inflammatory activities and PTUPB multiple organ protective actions are associated with strong antiinflammatory actions. However, it should be noted that in these earlier studies PTUPB treatment was used in preventive manner. In the present study PTUPB was used in interventional manner and it reduced renal inflammation in an event when disease was already well established and where inflammation is an important pathophysiological factor.

Anti-inflammatory actions of interventional PTUPB treatment are most likely caused by the inhibition of both COX-2 and sEH pathways, as inhibitors of each of these pathways have ability to reduce renal inflammation (Gassler et al., 2001; Bombardier et al., 2000). sEH inhibition demonstrated renal antiinflammatory action in hypertension and diabetes animal models. Global sEH knockout (EPHX2 -/-) mice treated with deoxycorticosterone acetate in combination with high salt (DOCA-salt) had lower inflammatory gene expression and lesser degree of renal macrophage infiltration compared to wild type mice (Manhiani et al., 2009). Moreover, in a renal fibrosis model, sEH inhibition either by gene knockout or by pharmacological inhibition provided antifibrotic action in the kidney by reducing renal inflammation (Kim et al., 2015). Not only sEH inhibition but also COX-2 inhibition demonstrated anti-inflammatory action in the kidney. In ZDF rats, the diabetic kidney injury associated with elevated renal inflammation is reduced by COX-2 inhibition (Dey et al., 2004). Indeed, several studies demonstrated marked anti-inflammatory actions of COX 2 inhibition in multiple renal pathologies including type 2 diabetes (Honma et al., 2013; Fujihara 
et L., 2003). In an earlier study, we demonstrated that COX-2 inhibitor rofecoxib reduced renal tubular glomerular injury in type 2 diabetic obese ZDF rats, and the renal action of rofecoxib was associated with its anti-inflammatory effect (Dey et al., 2004). These earlier findings led us to suggest that the anti-inflammatory actions of COX-2 and sEH inhibition contributed to the marked renal anti-inflammatory actions of PTUPB. Overall, we demonstrate a unique biological action of the dual acting sEH/COX-2 inhibitor PTUPB in treating renal inflammation and injury in a rat model with established renal dysfunction.

In diabetic nephropathy, along with tubular injury, glomerular injury is a pathophysiological hallmark of the kidney injury and dysfunction. We demonstrated that the type 2 diabetic obese ZSF1 rats had marked glomerular injury and damage in the glomerular filtration barrier as assessed from reduced expression of slit diaphragm component nephrin. Interventional PTUPB treatment markedly reduced renal injury in type 2 diabetic obese ZSF1 rats. In the preceding sections we have discussed the diabetic kidney injury treating ability of PTUPB in terms of its beneficial actions on metabolic dysfunctions such as hypertension, hyperlipidemia, and also marked renal inflammation in obese ZSF1 rats. Apart from these approaches, we further investigated renal action of PTUPB in an in vitro study using isolated glomeruli. We determined the ability of PTUPB in maintaining glomerular permeability, an important functional feature of glomeruli for their efficient filtration capacity. We demonstrated that PTUPB directly maintains normal glomerular permeability. The findings of this in vitrostudy suggest that the renal injury treating ability of PTUPB in diabetic nephropathy is not only caused by its ability to reduce renal inflammation but also due to its direct effects on the glomerular filtration barrier. Indeed, an important role of endogenous CYP450 metabolites of arachidonic acid in maintaining the glomerular protein permeability barrier has been reported. It has been shown that EETs play an important role in maintaining normal glomerular permeability (Williams et al., 2007). Increased COX-2 expression in podocytes also leads to increased glomerular permeability (Cheng et al., 2007). Thus, PTUPB likely decreases glomerular barrier injury through inhibitory actions on both sEH and COX-2.

Apart of diabetic nephropathy, in type 2 diabetes co-morbid conditions like hyperlipidemia and obesity often contribute to multiple organ injury. Type 2 diabetic patients with co-morbid hyperlipidemia are at high risk to develop chronic liver disease, particularly non-alcoholic fatty liver disease (NAFLD) (Younossi et al., 2019). In the present study, as well as in an earlier study, we demonstrated that diabetic nephropathy is accompanied by liver dysfunction and steatosis in type 2 diabetic obese ZSF1 rats (Hye Khan et al., 2018). Interestingly, interventional PTUPB but not enalapril treatment markedly reduced liver injury and steatosis in obese ZSF1 rats. Our findings are in accord with an earlier study which reported that the ACE inhibitor enalapril did not treat liver dysfunction and steatosis in obese ZSF1 rats (Bilan et al., 2011).

In regard to the action of PTUPB, a recent study demonstrated liver protective effect of PTUPB. It is shown that PTUPB reduced liver weight, liver lipid content, steatosis, and the liver expression of lipolytic/lipogenic and lipid uptake related genes in a high fat diet induced NAFLD mouse model. PTUPB treatment also arrested liver fibrosis with a decreased collagen deposition and expression of several fibrotic markers including $\alpha$-smooth muscle action (Sun et al., 2020). It is suggested that the effects of PTUPB on liver dysfunction particularly on hepatostetosis is associated with its lipid lowering and anti-inflammatory actions (Sun et al., 2020). The liver protective actions of PTUPB can also be related to the effect of sEH inhibition on the liver. sEH inhibitors reduced liver steatosis in a high-fat diet induced metabolic disease model (Liu et al., 2012). A strong anti-fibrotic action of sEH inhibitor is also reported in carbon tetrachloride induced cirrhotic hepatitis model (Harris et al., 2015). Activation of COX-2 is reported to be involve in liver injury, hence, COX-2 inhibitor will have a beneficial liver protective effect (Horrillo et al., 2007). The liver effects of PTUPB are also likely linked to the anti-inflammatory and lipid lowering actions. Overall, we demonstrated that the dual acting sEH/COX-2 inhibitor, PTUPB, has promising effects on liver dysfunction that is associated with type 2 diabetes and other similar metabolic disorders.

In summary, we tested a unique dual acting sEH/COX-2 inhibitor, PTUPB that given in an interventional manner is not anti-diabetic but can effectively treat diabetic renal injury and several co-morbid conditions in type 2 diabetes. Interventional PTUPB treatment has multiple actions including decreasing diabetic 
kidney injury, lowering blood pressure, and reducing hyperlipidemia. We further found that PTUPB has promising effect on type diabetes related liver disease. We believe the small molecule dual acting sEH/COX2 inhibitor PTUPB has promising drug development potential for diabetic nephropathy and other diabetic complications.

\section{FUNDING}

A National Institute of Health grant DK103616 (J.D.I.), Dr. Ralph and Marian Falk Medical Research Trust Bank of America, N.A., Trustee grant (J.D.I.), and PhRMA Foundation, Postdoctoral Fellowship (S.D.B.). Partial support was provided by the NIH-NIEHS (RIVER Award) R35 ES030443-01, and the NIEHS Superfund Research Program P42 ES004699 (B.D.H.).

\section{AUTHOR CONTRIBUTIONS}

Md.A.H.K. and J.D.I. contributed in the conception and design of the study, in the analysis and interpretation of the data, in drafting the article. Md.A.H.K., S.D.B., A.B., and W.K.J. caried out experiments and data analysis. S.H.H. and B.D.H. design and synthesized PTUPB. All authors critically reviewed the data, checked data interpretation and provided final approval of the version to be submitted.

\section{DECLARATION OF TRANSPARENCY AND SCIENTIFIC RIGOUR}

This Declaration acknowledges that this paper adheres to the principles for transparent reporting and scientific rigor of preclinical research as stated in the BJP guidelines for Design \& Analysis, Immunochemistry, and Animal Experimentation as recommended by funding agencies, publishers, and other organizations engaged with supporting research.

\section{DISCLOSURE}

The dual inhibitor is covered under a University of California Patent with Sung Hee Hwang and Bruce D. Hammock. Other authors declare no conflicts of interest, financial or otherwise.

\section{REFERECES}

1. Afkarian, M., Sachs, M.C., Kestenbaum, B., Hirsch, I. B., Tuttle, K.R., Himmelfarb, J. ... de Boer, I.H. (2013). Kidney disease and increased mortality risk in type 2 diabetes. Journal of American Society of Nephrology, 24, 302-308. doi:10.1681/ASN.2012070718

2. Batlle, D., Wysocki, J., Soler, M.J. (2012). Angiotensin-converting enzyme 2: enhancing the degradation of angiotensin II as a potential therapy for diabetic nephropathy. Kidney International ., 81, 520-528. doi:10.1038/ki.2011.381

3. Bellucci, P.N., González Bagnes, M.F., Di Girolamo, G., González, C.D. (2017). Potential Effects of Nonsteroidal Anti-Inflammatory Drugs in the Prevention and Treatment of Type 2 Diabetes Mellitus. Journal of Pharmacy Practice ., 30, 549-556. doi: 10.1177/0897190016649551

4. Bilan, V.P., Salah, E.M., Bastacky, S., Jones, H.B., Mayers, R.M., Zinker, B., Poucher, S.M., Tofovic, S.P. (2011). Diabetic nephropathy and long-term treatment effects of rosiglitazone and enalapril in obese ZSF1 rats. Journal of Endocrinology , 210, 293-308. doi: 10.1530/JOE-11-0122

5. Bombardier, C., Laine, L., Reicin, A., Shapiro, D., Burgos-Vargas, R., Davis, B., ... Schnitzer, T.J. (2000). Comparison of upper gastrointestinal toxicity of rofecoxib and naproxen in patients with rheumatoid arthritis, VIGOR Study Group. New England Journal of Medicine, 343, 1520-1528. doi: 10.1056/NEJM200011233432103

6. Cases, A., Coll, E. (2005). Dyslipidemia and the progression of renal disease in chronic renal failure patients. Kidney International, 99, S87-S93. doi: 10.1111/j.1523-1755.2005.09916.x

7. Catalá-López, F., Macías Saint-Gerons, D., González-Bermejo, D., Rosano, G.M.,... Hutton, B. (2016). Cardiovascular and Renal Outcomes of Renin-Angiotensin System Blockade in Adult Patients with Diabetes Mellitus: A Systematic Review with Network Meta-Analyses.PLoS Medicine, 13(3), e1001971. doi:10.1371/journal.pmed.1001971 
8. Chalikias, G., Tziakas, D. (2020). Angiotensin Receptor Neprilysin Inhibitors-2019 Update. Cardiovascular Drugs Ther apy, 10.1007/s10557-020-07015-8. doi:10.1007/s10557-020-07015-8

9. Cheng, H., Wang, S., Jo, Y-I., Hao, C-M., Zhang, M., Fan, X., ... Harris, R.C. (2007). Overexpression of cyclooxygenase-2 predisposes to podocyte injury. Journal of American Society of Nephrol ., 18, 551-559. doi: 10.1681/ASN.2006090990

10. Cheng, H.F., Harris, R.C. (2004). Cyclooxygenases, the kidney, and hypertension. Hypertension, 43, 525-530. doi: 10.1161/01.HYP.0000116221.27079.ea

11. Cho, J., Cooke, C.E., Proveaux, W. (2003). A retrospective review of the effect of COX-2 inhibitors on blood pressure change. American Journal of Therapy, 10, 311-317. doi:10.1097/00045391-20030900000002

12. Delanaye P, Scheen AJ. Preventing and treating kidney disease in patients with type 2 diabetes. Expert Opin Pharmacother . 2019;20(3):277-294. doi:10.1080/14656566.2018.1551362

13. Dey, A., Maric, C., Kaesemeyer, W.H., Zaharis, C.Z., Stewart, J., Pollock, J.S., Imig, J.D. (2004). Rofecoxib decreases renal injury in obese Zucker rats. Clinical Sciences (Lond) , 107, 561-570. doi: 10.1042/CS20040125.

14. EnayetAllah, A.E., Luria, A., Luo, B., Tsai, H-J., Sura, P., Hammock, B.D., Grant, D.F. (2008). Opposite Regulation of Cholesterol Levels by the Phosphatase and Hydrolase Domains of Soluble Epoxide Hydrolase. Journal of Biological Chemistry , 283, 36592-36598. doi: 10.1074/jbc.M806315200

15. Ferro, C.L., Mark, P.B., Kanbay, M., Sarafidis, P., Heine, G.H., Rossignol, P.,... Zoccali, C. (2018). Lipid management in patients with chronic kidney disease Nature Review of Nephrology, 14, 727-749. doi: 10.1038/s41581-018-0072-9

16. Fujihara, C.K., Antunes, G.R., Mattar, A.L., Andreoli, N., Malheiros D.M.A.C., .. Zatz, R. (2003). Cyclooxygenase-2 (COX-2) inhibition limits abnormal COX-2 expression and progressive injury in the remnant kidney. Kidney International , 64, 2172-2181. doi: 10.1046/j.1523-1755.2003.00319.x

17. Furman, D., Campisi, J., Verdin, E., Carrera-Bastos, P., Targ, S., ...Slavich, G.M. (2019). Chronic inflammation in the etiology of disease across the life span. Nature Medicine , 25, 1822-1832. doi: 10.1038/s41591-019-0675-0

18. Gartung, A., Yang, J., Sukhatme, V.P.,Bielenberg, D.R.,...Panigrahy, D. (2019). Suppression of chemotherapy-induced cytokine/lipid mediator surge and ovarian cancer by a dual COX-2/sEH inhibitor. Proceedings of National Academy of Sciences USA , 116, 1698-1703. doi:10.1073/pnas.1803999116

19. Gassler, N., Elger, M., Kränzlin, B., Kriz, W., Gretz, N., ...Hartmann, I. (2001). Podocyte injury underlies the progression of focal segmental glomerulosclerosis in the fa/fa Zucker rat, Kidney International, 60, 106-116. doi: 10.1046/j.1523-1755.2001.00777.x

20. Grosser, T., Fries, S., FitzGerald, G.A. (2006). Biological basis for the cardiovascular consequences of COX-2 inhibition: therapeutic challenges and opportunities. Journal of Clinical Investigation, 116, 4-15. doi:10.1172/JCI27291

21. Grundy, S.M. (2006). Drug Therapy of the Metabolic Syndrome: Minimizing the Emerging Crisis in Polypharmacy. Nature Review of Drug Discovery, 5, 295-309. doi: 10.1038/nrd2005

22. Harris, R.C. (2008). An update on cyclooxygenase-2 expression and metabolites in the kidney. Current Opinion of Nephrology and Hypertension , 17, 64-69. doi: 10.1097/MNH.0b013e3282f1bb7d

23. Harris, T.R., Bettaieb, A., Kodani, S., Dong, H., Myers, R., ... Hammock, B.D. (2015) Inhibition of soluble epoxide hydrolase attenuates hepatic fibrosis and endoplasmic reticulum stress induced by carbon tetrachloride in mice. Toxicology and Applied Pharmacology, 286,102-111. doi: 10.1016/j.taap.2015.03.022

24. Homer, B.L., Dower, K. (2018). 41-Week Study of Progressive Diabetic Nephropathy in the ZSF1 fa/fa ${ }^{\mathrm{CP}}$ Rat Model. Toxicology and Pathology , 46, 976-977. doi: 10.1177/0192623318803278

25. Horrillo, R., Planagumà, A., González-Périz, A., Ferré, N.,... Clària, J. (2007). Comparative protection against liver inflammation and fibrosis by a selective cyclooxygenase- 2 inhibitor and a nonredoxtype 5-lipoxygenase inhibitor. Journal of Pharmacology and Experimental Therapeutics, 323, 778-786. doi:10.1124/jpet.107.128264

26. Hotamisligil, G.S. (2006). Inflammation and metabolic disorders.Nature , 444, 860-867. doi: 
$10.1038 /$ nature 05485

27. Hye Khan, M.A,. Hwang, S.H., Sharma, A., Corbett, J.A., ... Imig, J.D. (2016). A dual COX-2/sEH inhibitor improves the metabolic profile and reduces kidney injury in Zucker diabetic fatty rat. Prostaglandins and Other Lipid Mediators , 125, 40-47. doi: 10.1016/j.prostaglandins.2016.07.003

28. Hye Khan, M.A., Kolb, L., Skibba, M., Hartmann, M., Blöcher, R.,... Imig, J.D. (2018). A novel dual PPAR- $\gamma$ agonist/sEH inhibitor treats diabetic complications in a rat model of type 2 diabetes. Diabetologia , 61, 2235-2246. doi: 10.1007/s00125-018-4685-0

29. Hye Khan, M.A., Schmidt, J., Stavniichuk, A., Imig, J.D., Merk, D. (2019). A dual farnesoid X receptor/soluble epoxide hydrolase modulator treats non-alcoholic steatohepatitis in mice. Biochemical Pharmacology , 166, 212-221. doi: 10.1016/j.bcp.2019.05.023

30. Ilatovskaya, D.V., Palygin, O., Levchenko, V., Endres, B.T., Staruschenko, A. (2017). The Role of Angiotensin II in Glomerular Volume Dynamics and Podocyte Calcium Handling. Scientific Reports , 7, 299. doi: 10.1038/s41598-017-00406-2

31. Imig, J.D. Walsh, K.A., Hye Khan, M.A., Nagasawa, T., Cherian-Shaw, M., .. Hammock, B.D. (2012) Soluble epoxide hydrolase inhibition and peroxisome proliferator activated receptor $\gamma$ agonist improve vascular function and decrease renal injury in hypertensive obese rats. Experimental Biology and Medicine (Maywood) , 237, 1402-1412. doi: 10.1258/ebm.2012.012225

32. Imig, J.D. (2018). Prospective for cytochrome P450 epoxygenase cardiovascular and renal therapeutics. Pharmacology and Therapeutics, 192, 1-19. doi: 10.1016/j.pharmthera.2018.06.015

33. Imig, J.D., Zhao, X., Capdevila, J.H., Morisseau, C., Hammock, B.D. (2002). Soluble epoxide hydrolase inhibition lowers arterial blood pressure in angiotensin II hypertension. Hypertension . 39(2002) 690694. doi: 10.1161/hy0202.103788

34. Imig, J.D., Zhao, X., Zaharis, C.Z., Olearczyk, J.J., Pollock, D.M., ... Hammock, B.D. (2005). An orally active epoxide hydrolase inhibitor lowers blood pressure and provides renal protection in saltsensitive hypertension. Hypertension, 46, 975-981

35. Isomaa, B., Almgren, P., Tuomi, T., Forsén, B., Lahti, K.,.. . Groop, L. (2001). Cardiovascular morbidity and mortality associated with the metabolic syndrome. Diabetes Care , 24, 683-689. doi: 10.2337/diacare.24.4.683

36. Kim, J., Yoon, S.P., Toews, ML, Imig, J.D., Hwang, S.H., ...Padanilam, B. (2015). Pharmacological inhibition of soluble epoxide hydrolase prevents renal interstitial fibrogenesis in obstructive nephropathy. American Journal of Physiology Renal Physiology, 308, F131-F139. doi: 10.1152/ajprenal.00531.2014

37. Liu, Y., Dang, H., Li, D., Pang, W., Hammock, B.D., Zhu, Y. (2012). Inhibition of Soluble Epoxide Hydrolase Attenuates High-Fat-Diet-Induced Hepatic Steatosis by Reduced Systemic Inflammatory Status in Mice. PLoS ONE , 7, e39165. doi: 10.1371/journal.pone.0039165

38. Lorthioir, A., Guerrot, D., Joannides, R., Bellien, J. (2012). Diabetic CVD-soluble epoxide hydrolase as a target. Cardiovascular 83 Hematological Agents in Medicinal Chemistry, 10, 212-222. doi: $10.2174 / 187152512802651042$

39. Ma, S., Zhu, X-Y., Eirin, A., Woollard, J.R. Jordan, K.L., . . Lerman, L.O. (2016) Perirenal fat promotes renal arterial endothelial dysfunction in obese swine 508 through tumor necrosis factor-alpha. Journal of Urolpgy, 195, 1152-1259. doi: 10.1016/j.juro.2015.08.105

40. Manhiani, M., Quigley, J.E., Knight, S.F., Tasoobshirazi, S.T., Moore, T., . . Imig, J.D. (2009). Soluble epoxide hydrolase gene deletion attenuates renal injury and inflammation with DOCA-salt hypertension. American Journal of Physiology Renal Physiology,297, F740-F748. doi: 10.1152/ajprenal.00098.2009

41. Nandakishore R., Yalavarthi, P.R., Kiran, Y.R., Rajapranathi, M. (2014). Selective cyclooxygenase inhibitors: current status.Current Drug Discovery and Technology , 11, 127-132. doi:10.2174/1570163811666140127123717

42. Nasrallah, R., Hassouneh, R., Hébert, R.L. (2016). PGE2, Kidney Disease, and Cardiovascular Risk: Beyond Hypertension and Diabetes. Journal of American Society of Nephrology, 27, 666-676. doi: 10.1681/ASN.2015050528

43. Neckáŕ. J., Kopkan. L., Husková, Z., Kolář, F., Papoušek, F.,.. Červenka, L. (2012). Inhibition 
of soluble epoxide hydrolase by cis-4-[4-(3-adamantan-1-ylureido)cyclohexyl-oxy]benzoic acid exhibits antihypertensive and cardioprotective actions in transgenic rats with angiotensin II-dependent hypertension. Clinical Science (London), 122, 513-525. doi: 10.1042/CS20110622

44. Patti, A.M., Rizvi, A.A., Giglio, R.V., Stoian, A.P., Ligi, D., Mannello, F. (2020). Impact of GlucoseLowering Medications on Cardiovascular and Metabolic Risk in Type 2 Diabetes. Journal of Clinical Medicine, 9, 912. doi:10.3390/jcm9040912

45. PTUPB ameliorates high-fat diet-induced non-alcoholic fatty liver disease via inhibiting NLRP3 inflammasome activation in mice. Biochemical and Biophysical Research Communications , 523, 1020-1026. doi: $10.1016 /$ j.bbrc.2019.12.131

46. Ritz, E., Tarng, D.C. (2001). Renal disease in type 2 diabetes. Nephrology Dialysis and Transplantation, 5, 11-18. doi: 10.1093/ndt/16.suppl_5.11

47. Roche, C., Guerrot, D., Harouki, N., Duflot, T., Besnier, M.,... Bellien, J. (2015). Impact of soluble epoxide hydrolase inhibition on early kidney damage in hyperglycemic overweight mice. Prostaglandins and Other Lipid Mediators, 120, 148-154. doi: 10.1016/j.prostaglandins.2015.04.011

48. Rosenthal, T., Erlich, Y., Rosenmann, E., Grossman, E., Cohen, A. (1995). Enalapril improves glucose tolerance in two rat models: a new hypertensive diabetic strain and a fructose-induced hyperinsulinaemic rat. Clinical and Experimental Pharmacology and Physiology,1995;22(1):S353-S354. doi: 10.1111/j.1440-1681.1995.tb02951.x

49. Safaeian, L., Hajhashemi, V., Ajoodanian, M. (2018) The effect of celecoxib on blood pressure and plasma oxidant/antioxidant status in co-administration with glucocorticoid in rat. Biomed Pharmacother , 108, 1804-1808. doi:10.1016/j.biopha.2018.10.047

50. Saran, R., Robinson, B., Abbott, K.C., Bragg-Gresham, J., Xiaoying Chen, X.,... Shahinian, V. (2020). US Renal Data System 2019 Annual Data Report: Epidemiology of Kidney Disease in the United States. American Journal of Kidney Disease, 75, A6-A7. doi: 10.1053/j.ajkd.2019.09.003

51. Su, Z., Widomski, D., Nikkel, A., Leys, L...,. McGaraughty, S. (2018). Losartan improves renal function and pathology in obese ZSF-1 rats. Journal of Basic and Clinical Physiologyl Pharmacoogy,9(3), 281-290. doi:10.1515/jbcpp-2017-0157

52. Toth-Manikowski, S., Atta, M.G. (2015). Diabetic Kidney Disease: Pathophysiology and Therapeutic Targets. Journal of Diabetes Research, . 2015, 697010. doi: 10.1155/2015/697010

53. Vuorinen-Markkola, H., Yki-Järvinen, H. (1995). Antihypertensive therapy with enalapril improves glucose storage and insulin sensitivity in hypertensive patients with non-insulin-dependent diabetes mellitus. Metabolism , 44, 85-89. doi: 10.1016/0026-0495(95)90293-7

54. Warren, A.M., Knudsen, S.T., Cooper, M.E. (2019). Diabetic nephropathy: an insight into molecular mechanisms and emerging therapies. Expert Opinion in Therapeutic Targets, 23, 579-591. doi: 10.1080/14728222.2019.1624721

55. Williams, J.M., Sharma, M., Anjaiahh, S., Falck, J.R., Roman, R.J. (2007). Role of endogenous CYP450 metabolites of arachidonic acid in maintaining the glomerular protein permeability barrier. American Journal of Physiology and Renal Physiology,293, F501-F505. doi: 10.1152/ajprenal.00131.2007

56. Xie X, Atkins E, Lv J, Bennett, A.,... Rodgers, A. (2016). Effects of intensive blood pressure lowering on cardiovascular and renal outcomes: updated systematic review and meta-analysis. Lancet , 387, 435-443. doi:10.1016/S0140-6736(15)00805-3

57. Younossi, Z.M., Henry, L. (2019). The Impact of Obesity and Type 2 Diabetes on Chronic Liver Disease. American Journal of Gastroenterology, 114, 1714-1715. doi: 10.14309/ajg.0000000000000433

58. Zhang, X., Lerman, L.O. (2016). The metabolic syndrome and chronic kidney disease. Translational Research, 183, 14-25. doi: 10.1016/j.trsl.2016.12.004

59. Zhang, Y.F., Sun, C.C., Duan, J.X., Yang, H.H.,...Guan, C.X. (2020). A COX-2/sEH dual inhibitor PTUPB ameliorates cecal ligation and puncture-induced sepsis in mice via anti-inflammation and anti-oxidative stress. Biomeddical Pharmacotherapy , 126, 109907. doi:10.1016/j.biopha.2020.109907

60. Zhao, X., Dey, A., Romanko, O.P., Stepp, D.W., Wang, H.H., ... Imig, J.D. (2005). Decreased epoxygenase and increased epoxide hydrolase expression in the mesenteric artery of obese Zucker rats. American Journal of Physiology Regulatory Integrative and Comparative Physiology , 288, R188-R196. doi: 


\subsection{2/ajpregu.00018.2004}

\section{FIGURE LEGEND}

Figure 1 : Diabetic parameters in experimental groups.Fasting blood glucose (A), hemoglobin $A_{1 c}$ (B), serum insulin level (C) and HOMA Index at the end of the 8-week experimental protocol. ${ }^{*} \mathrm{p}<0.05$ vs. lean ZSF1-Vehicle; $++\mathrm{p}<0.05$ vs. obese ZSF1-Vehicle. ENAL $=$ enalapril. Data are reported as box and whisker plots with median, minimum to maximum, and 10 to 90 percentiles, $n=6 /$ group.

Figure 2 : Circulating lipids in experimental groups.Plasma levels of cholesterol (A), non-esterified free fatty acid (B), triglyceride (C), and LDL-cholesterol (D) at the end of the 8-week experimental protocol. ${ }^{*} \mathrm{p}<0.05$ vs. lean ZSF1-Vehicle; $++\mathrm{p}<0.05$ vs. obese ZSF1-Vehicle. ENAL $=$ enalapril. Data are reported as box and whisker plots with median, minimum to maximum, and 10 to 90 percentiles, $\mathrm{n}=6$ /group.

Figure 3 : Renal injury in experimental groups. Calculated values of renal cortical (A) and medullary (B) cast area at the end of the 8-week experimental protocol. Albuminuria (C) and a representative photomicrograph showing tubular cast in the renal cortex and medulla (arrows) of different experimental groups analyzed at the end of the protocol (D). ${ }^{*} \mathrm{p}<0.05$ vs. lean ZSF1-Vehicle; $++\mathrm{p}<0.05$ vs. obese ZSF1-Vehicle. ENAL = enalapril. Data are reported as box and whisker plots with median, minimum to maximum, and 10 to 90 percentiles, $n=6$ /group.

Figure 4: Glomerular injury in experimental groups.Calculated values of glomerular injury score (A) and glomerular nephrin expression (C) at the end of the 8-week experimental protocol. Representative photomicrograph showing damaged glomeruli (arrows) (B) and nephrin expression (arrows) in the glomeruli of different experimental groups analyzed at the end of the protocol (D). ${ }^{*} \mathrm{p}<0.05$ vs. lean ZSF1-Vehicle; $++\mathrm{p}<0.05$ vs. obese ZSF1-Vehicle. ENAL $=$ enalapril. Data are reported as box and whisker plots with median, minimum to maximum, and 10 to 90 percentiles, $\mathrm{n}=6$ /group. Dual acting sEH-COX-2 inhibitor PTUPB attenuated angiotensin II (ANG II)-induced glomerular albumin permeability (E). *p $<0.05$ vs. Baseline; $++\mathrm{p}<0.05$ vs. ANG II-PTUPB. Data for E are expressed as mean + - SEM, $\mathrm{n}=10 /$ group

Figure 5 : Kidney inflammation in experimental groups.Urinary excretion of MCP-1 (A), CD-68 positive inflammatory cells in the kidney (B), and representative photomicrograph depicting CD-68 positive cells in the kidney $(\mathrm{C})$ at the end of the 8 -week experimental protocol. ${ }^{*} \mathrm{p}<0.05$ vs. lean ZSF1-Vehicle; $++\mathrm{p}<0.05$ vs. obese ZSF1-Vehicle. ENAL $=$ enalapril. Data are reported as box and whisker plots with median, minimum to maximum, and 10 to 90 percentiles, $n=6$ /group.

Figure 6 : Kidney fibrosis in experimental groups.Calculated values of renal cortical (A), medullary (B) collagen positive fibrotic area, and a representative photomicrograph showing renal fibrosis (C) at the end of the 8-week experimental protocol. ${ }^{*} \mathrm{p}<0.05$ vs. lean ZSF1-Vehicle; $++\mathrm{p}<0.05$ vs. obese ZSF1-Vehicle. $\mathrm{ENAL}=$ enalapril. Data are reported as box and whisker plots with median, minimum to maximum, and 10 to 90 percentiles, $n=6 /$ group.

Figure 7 : Liver injury in experimental groups. Plasma levels of AST (A), ALT (B), calculated value of liver collagen positive area $(\mathrm{C})$ and representative photomicrograph depicting collagen positive area (arrows) in the liver (E) at the end of the 8-week experimental protocol. Calculated values of oil o red positive (steatosis) liver area (D) and a representative photomicrograph liver steatosis (arrows) obtained from oil o red staining at the end of the 8-week experimental protocol (F). ${ }^{*} \mathrm{p}<0.05$ vs. lean ZSF1-Vehicle; $++\mathrm{p}<0.05$ vs. obese ZSF1-Vehicle. ENAL $=$ enalapril. Data are reported as box and whisker plots with median, minimum to maximum, and 10 to 90 percentiles, $n=6 /$ group. 
Figure 1

A

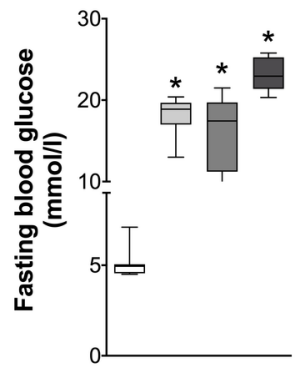

B

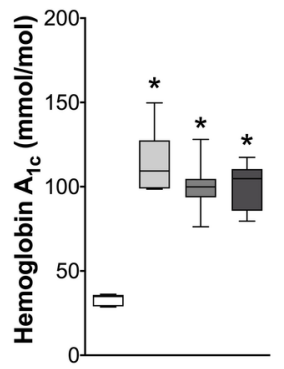

Figure 2

A

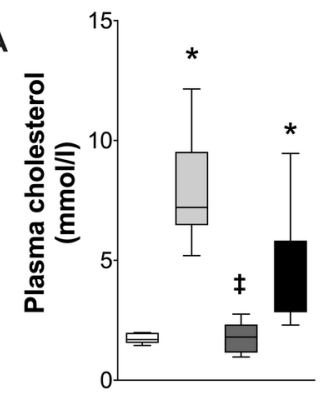

C

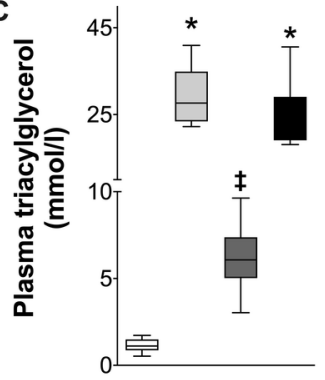

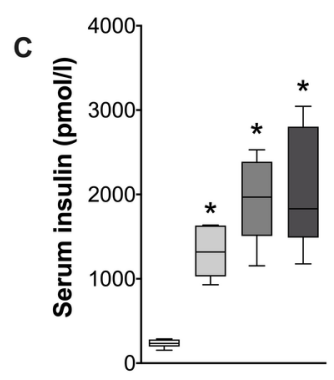

D

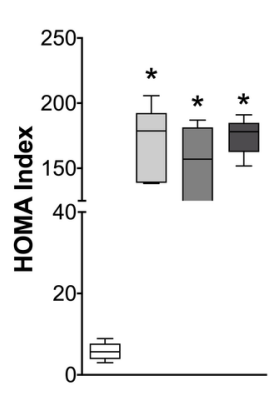

Lean ZSF1-Vehicle

Obese ZSF1-Vehicle

Obese ZSF1-PTUPB

Obese ZSF1-ENAL

Lean ZSF1-Vehicle

Obese ZSF1-Vehicle

Obese ZSF1-PTUPB

Obese ZSF1-ENAL

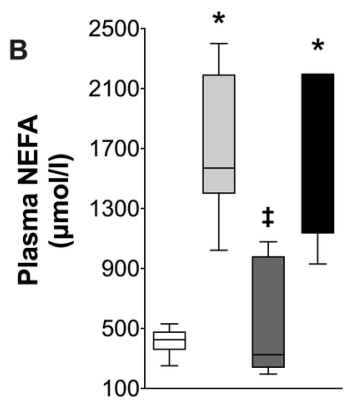

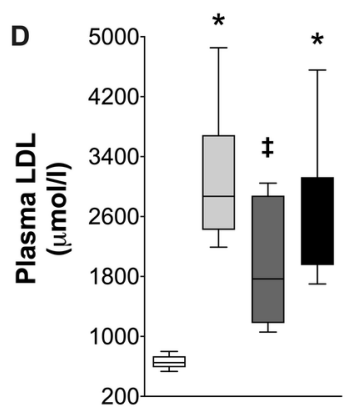




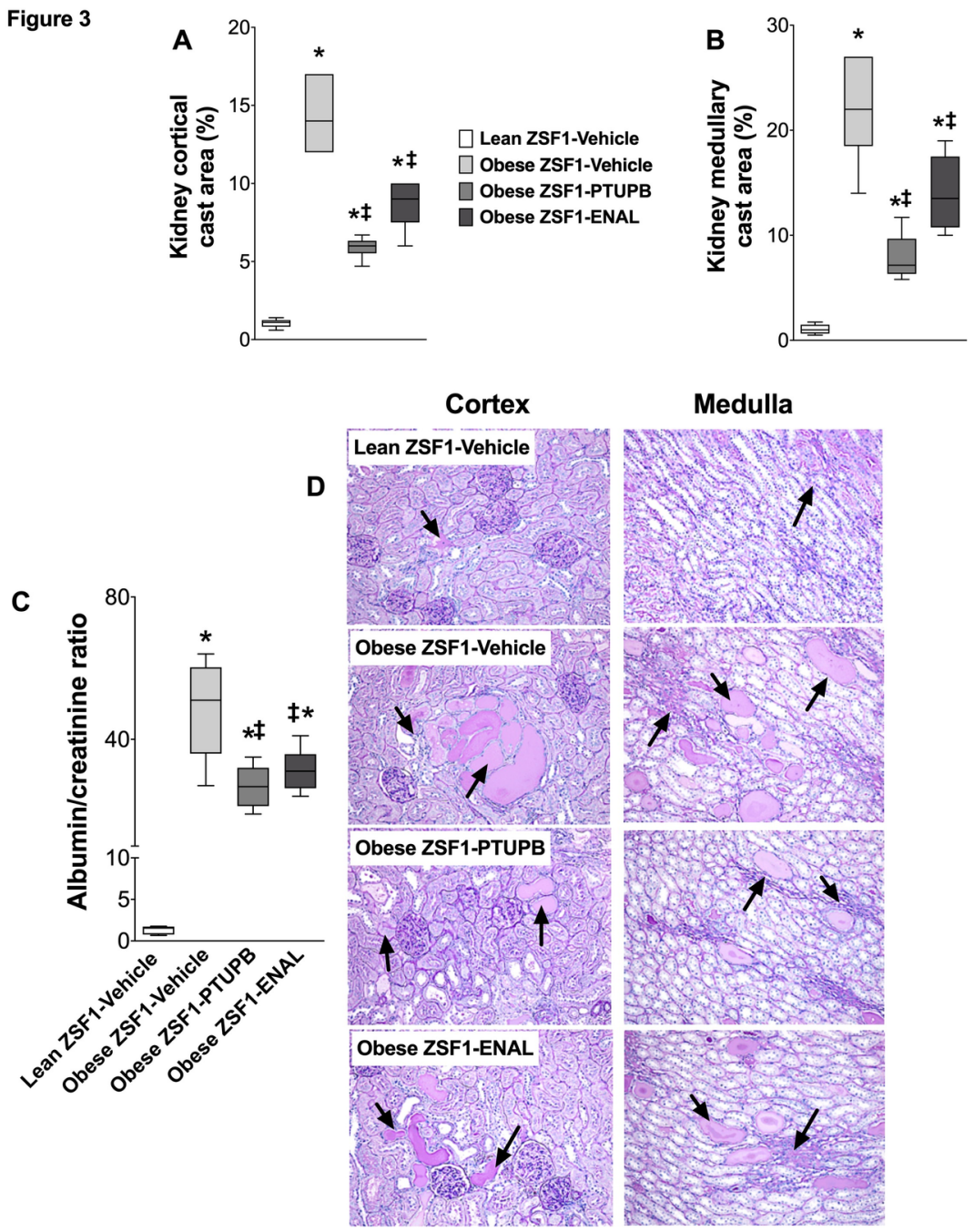


Figure 4
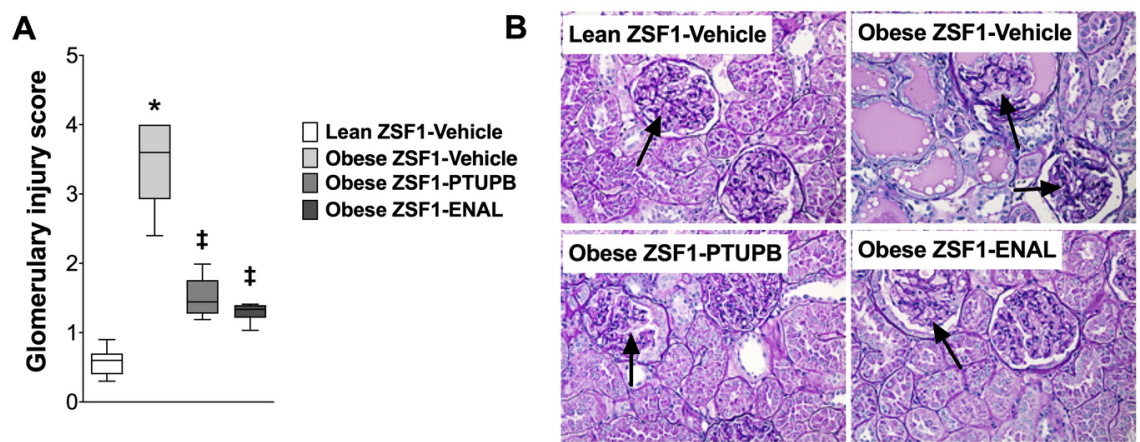

C

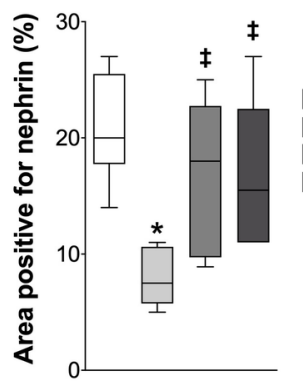

D

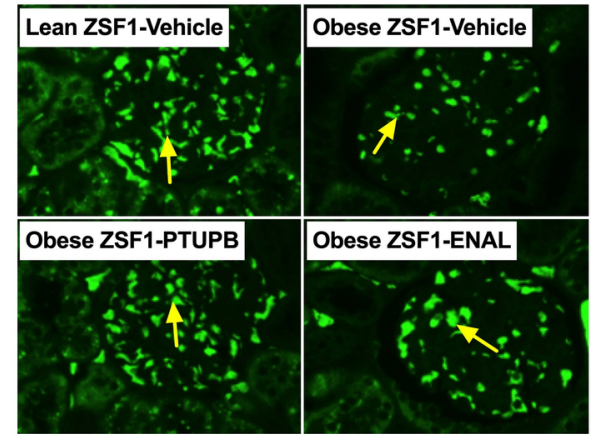

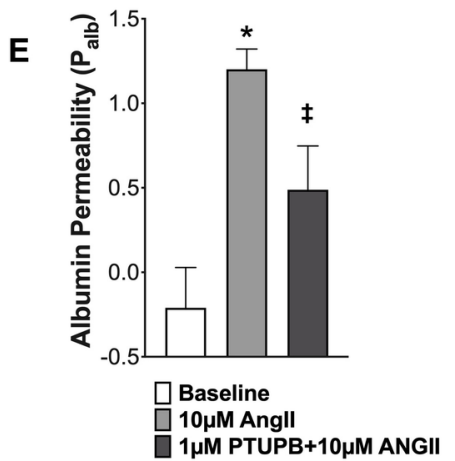


Figure 5

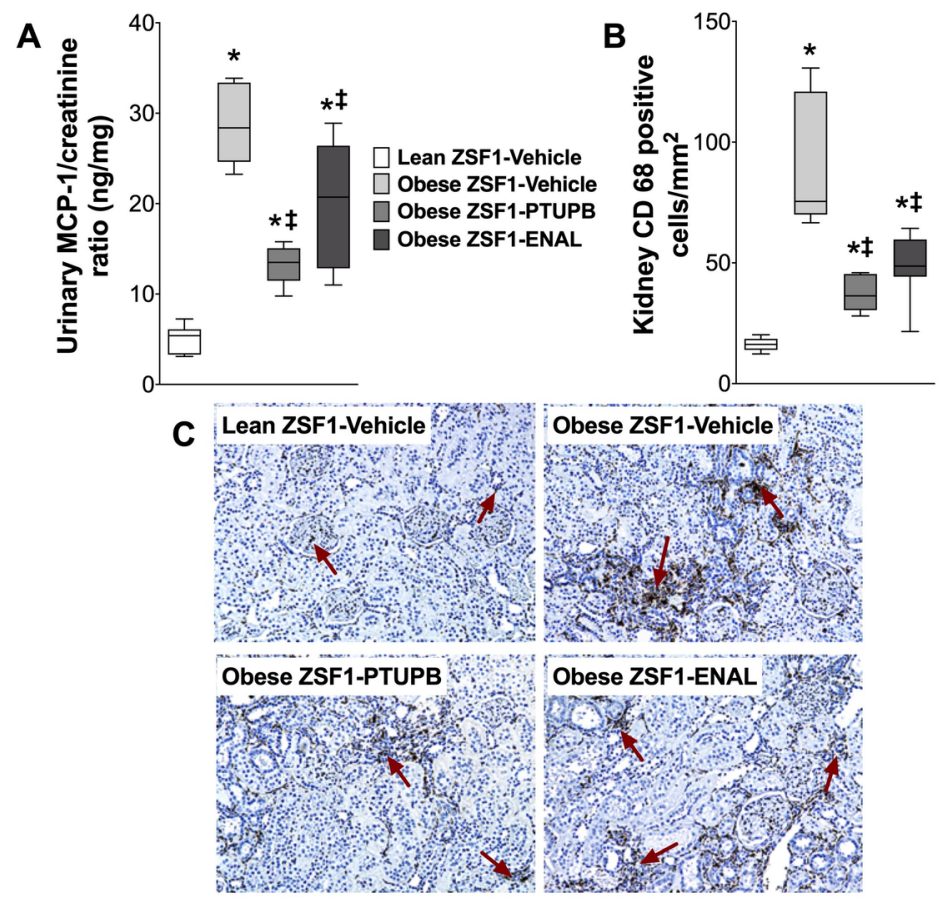

Figure 6

A

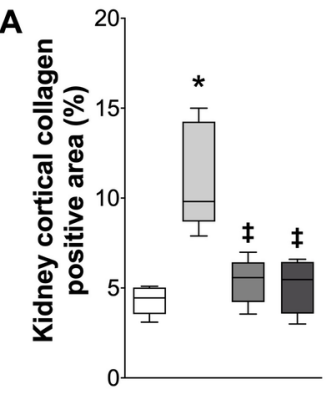

C Lean ZSF1-Vehicle

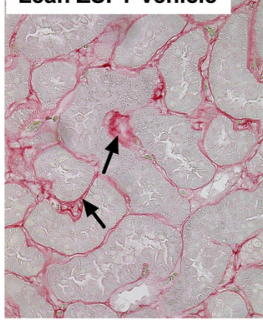

Lean ZSF1-Vehicle

$\square$ Obese ZSF1-Vehicle

$\square$ Obese ZSF1-PTUPB

Obese ZSF1-ENAL
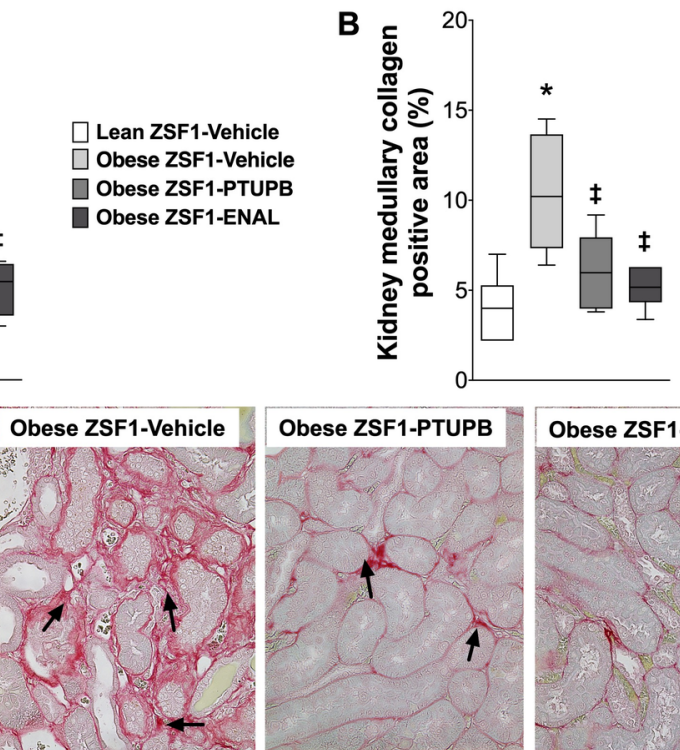

Obese ZSF1-PTUPB

Obese ZSF1-ENAL
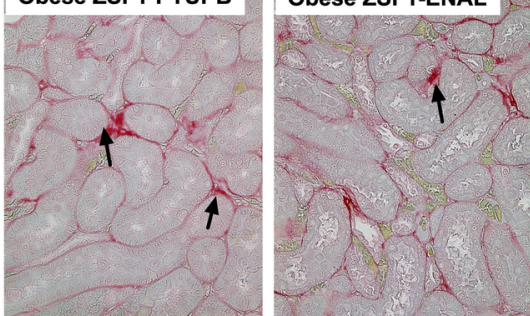
Figure 7
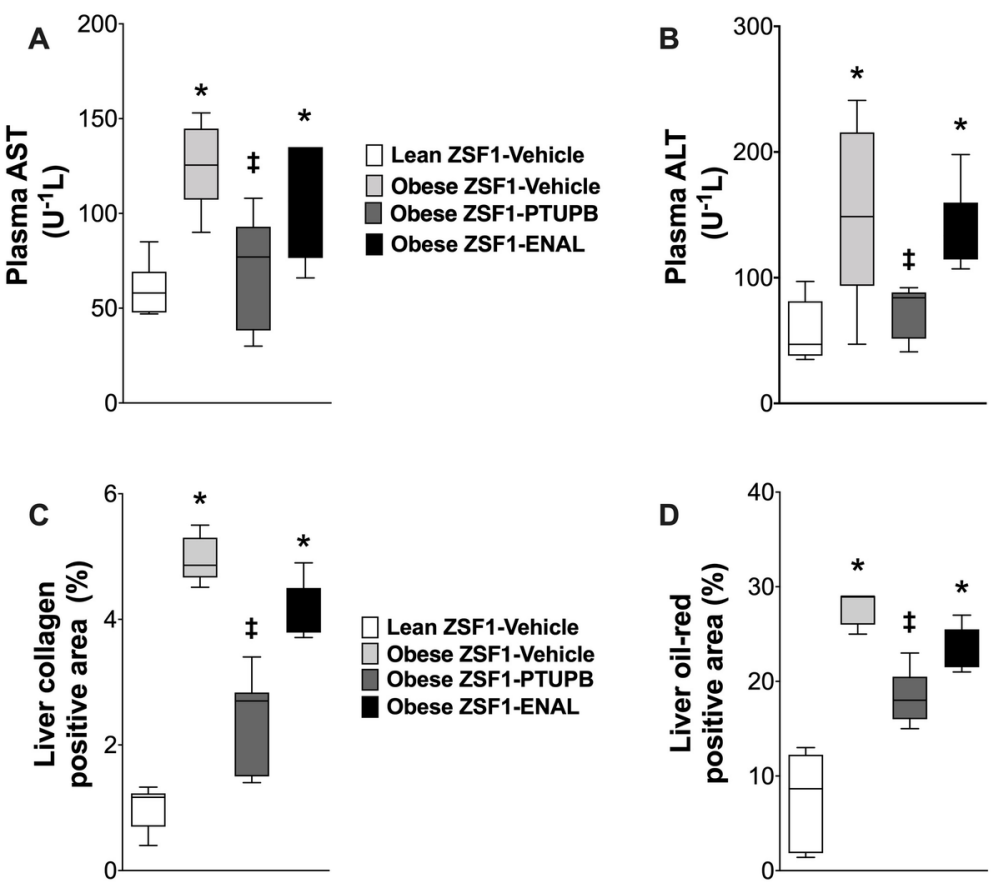

E

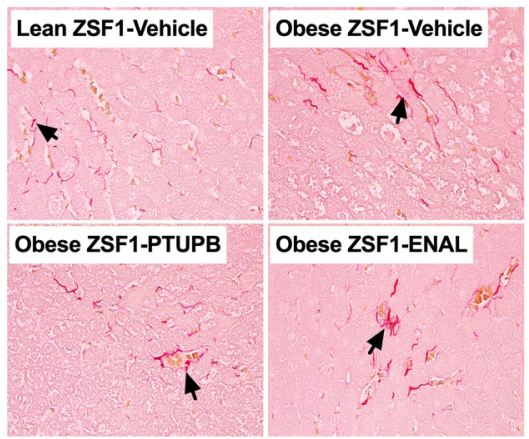

F

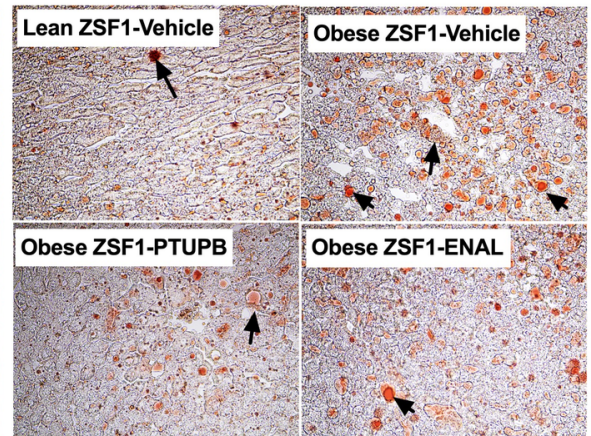

